\title{
Auxiliary Stopping Area Layout Method for High-Speed Maglev considering Multiple Speed Profiles and Bidirectional Operation
}

\author{
Danyang Dong $\mathbb{D}^{1,2}$ Yi Yu ${ }^{1},{ }^{1}$ Huahua Zhao, ${ }^{1}$ Pengzi Chu ${ }^{D},{ }^{1}$ and Hui Lin ${ }^{1}$ \\ ${ }^{1}$ Maglev Transportation Engineering R\&D Center, Tongji University, Shanghai 201804, China \\ ${ }^{2}$ College of Electronic and Information Engineering, Tongji University, Shanghai 201804, China \\ Correspondence should be addressed to Yi Yu; yuyi1962@tongji.edu.cn
}

Received 20 July 2021; Accepted 26 October 2021; Published 23 November 2021

Academic Editor: Yuan Gao

Copyright ( $\odot 2021$ Danyang Dong et al. This is an open access article distributed under the Creative Commons Attribution License, which permits unrestricted use, distribution, and reproduction in any medium, provided the original work is properly cited.

Auxiliary stopping area (ASA) is the key guarantee for high-speed maglev train operation safety. Aiming at the layout of the ASA along the track, this article constructs an optimized model of ASA layout and proposes a solving algorithm based on the single target genetic algorithm by analyzing the influence factors of ASA layout. The result of the numerical experiment shows that the proposed method could fulfill the requirements of the maglev train operation safety and efficiency and optimize the cost of the ASA layout while taking the complex line situation, train tracking operation, and bidirectional operation on a single line into account.

\section{Introduction}

With the continuous development of the economy and society, resource and environmental issues have become increasingly severe, and people hope to reduce their impact on the environment while traveling efficiently. Compared with traditional wheel-rail railways, the high-speed maglev transportation system could not only achieve higher speed but also have the advantages of low noise, low energy consumption, strong climbing ability, small turning radius, flexible route selection, easy maintenance, and high safety factor [1-3]. The development of high-speed maglev has attracted much attention, and countries such as China, Germany, and Japan have all carried out the development of high-speed maglev.

Due to different train operating mechanisms, there are many unique elements in maglev transportation. Among them, the auxiliary stopping area with charging rails and evacuation passages is an important safety characteristic for normal high-speed maglev transportation systems [4]. The operating maglev train should be able to stop in the auxiliary stopping area under any operational disturbances, failures, and emergency situations. If necessary, passengers should be able to evacuate from the train safely. At the same time, in order to maximize the guarantee that the train can stop in the auxiliary stopping area at any time, the operation of the maglev train adopts the stopping point stepping mode; that is, the current ASA is always the target point of operation during the stepping process of the train [5-10]. As the basis of the two-dimensional speed protection mode and the stopping point stepping mode, the layout of the auxiliary stopping area is related to the safety of train operation, operation efficiency, and construction cost [11]. The scientific method of arranging the auxiliary stopping area is worthy of discussion.

There is currently no exact reference standard or specification for the layout of auxiliary stopping areas. The engineering experience that can be referred to for high-speed maglev construction comes from the Shanghai Maglev Demonstration Line, which determines the length of the auxiliary stopping area according to the slope and the length of the vehicle, and delimits the reference distance between the auxiliary stopping areas according to the speed degree and slope [12]. This kind of auxiliary stopping area layout 
based on the nominal value is difficult to deal with complex working conditions, and the reference speed range is limited $(200-430 \mathrm{~km} / \mathrm{h})$. In recent years, with the deepening of highspeed maglev research, the research on the method of auxiliary stopping area layout has also attracted the attention of scholars.

Yu et al. [10] proposed a basic calculation method for the auxiliary stopping area layout of unidirectional track based on the safety protection speed profile. This method is a recursive arrangement strategy that takes the terminal station as the first reference auxiliary stopping area and then approaches the departure station one by one according to the protection speed profile. Later, they proposed a bidirectional coordinated auxiliary stopping area layout method for the single-track two-way line [13]. This method also takes a certain station as the reference station and operates an iterative process to arrange the auxiliary stopping area. Jiang et al. [14] proposed a method for the optimization and adjustment of the auxiliary stopping area layout, considering the operation scenario of the train tracking interval requirements. Lai et al. [15] set the minimum length of the auxiliary stopping area and the total length of the auxiliary stopping area as constraints and optimized the auxiliary stopping area layout of the medium- and high-speed maglev system from two aspects: the available area of speed and the Euclidean distance between the switching point of the maximum limit speed and the intersection of the braking curve and the levitation curve. Tian et al. [16] proposed a method for auxiliary stopping areas layout for the bidirectional track. Compared with arranging auxiliary stopping areas in two directions separately, it effectively reduces the number of auxiliary stopping areas required.

The operation of the maglev system often involves multiple speed profiles, and the actual working scenarios of the track, train, and environment are often more complicated. For instance, many track sections are not suitable for setting auxiliary stopping areas, and some sections need to set at least one auxiliary stopping area, which are designed for the convenience and actual needs of an emergency evacuation. At the same time, the layout of the auxiliary stopping area is related to the train tracking interval. If the operating efficiency of the train is considered, it is necessary to optimize the layout of the auxiliary stopping area according to this demand. Nevertheless, none of the existing studies has solved the above-mentioned problems well; that is to say, the method of the auxiliary stopping area layout is not perfect yet. In this regard, focusing on the safety of train operation, the construction cost of the line, and the operation efficiency of the train, this paper proposes a method for the layout of the high-speed maglev auxiliary stopping area. Specifically, through the description of the factors affecting the layout of the auxiliary stopping area, we established an optimization model of the auxiliary stopping area layout for multiple train operation speed profiles and single-track bidirectional operation scenarios and designed a genetic algorithm to solve the model according to the characteristics of the problem.

\section{Auxiliary Stopping Area Layout Model}

The normal high-speed maglev train operates in a "stopping point stepping" mode to ensure that it can stop in the auxiliary stopping area as much as possible. Specifically, the train always takes the current auxiliary stopping area as the target stopping point during the continuous stepping process and takes the maximum speed profile, the minimum speed profile, and the interval speed limit as the safety protection requirements. Only when it crosses the minimum speed profile of the next auxiliary stopping area and does not exceed the maximum speed profile of the current auxiliary stopping area, executing stepping, then the train sets the next auxiliary stopping area as the new target stopping point when it continues running, as shown in Figure 1.

2.1. Maximum and Minimum Speed Profile. The maximum and minimum speed profiles protect the train from reaching the safe braking profile and safe levitation profile. Therefore, the calculation of the maximum and minimum speed profiles is related to the safe braking profile and safe levitation profile. According to the dynamics model of the high-speed maglev train [17], the formula of the speed profile can be obtained. The calculation of the safe braking profile takes the force characteristics under adverse conditions into account. The adverse conditions include the full load of the train, the downwind effect, the partial failure of the eddy current braking system, and the decrease in the friction coefficient between the track guide-way and the train skid. Based on the force characteristics of the trains under the considered adverse conditions, there is a safe braking profile as in

$$
\left\{\begin{array}{l}
v_{t+1}^{b}=v_{t}^{b}+a_{t b}^{w} * \Delta t \\
s_{t+1}^{b}=s_{t}^{b}+v_{t}^{b} * \Delta t+\frac{a_{t b}^{w} * \Delta t^{2}}{2},
\end{array}\right.
$$

where $v_{t}^{b}$ is the velocity component at time $t$ of the safe braking profile; $s_{t}^{b}$ is the position component at time $t$ of the safe braking profile; $a_{t b}^{w}$ is the acceleration at time $t$ of the vehicles under adverse conditions; and $\Delta t$ is the sampling interval.

According to the safe braking profile, further considering the system delay $\Delta t_{b}$ from issuing the traction cutoff command to the completion of eddy current braking, speed measurement error $\Delta v$, positioning error $\Delta s$, and the maximum acceleration $a_{t b}^{w b}$ of the train during the time $\Delta t_{b}$, the maximum speed profile is calculable as in

$$
\left\{\begin{array}{l}
v_{t}^{\max }=v_{t}^{b}-a_{t b}^{w b} * \Delta t_{b}-\Delta v, \\
s_{t}^{\max }=s_{t}^{b}-\frac{a_{t b}^{w b} * \Delta t_{b}^{2}}{2}-v_{t}^{\max } * \Delta t_{b}-\Delta s,
\end{array}\right.
$$

where $v_{t}^{\max }$ is the velocity component of the maximum speed profile and $s_{t}^{\max }$ is the position component of the maximum speed profile. 


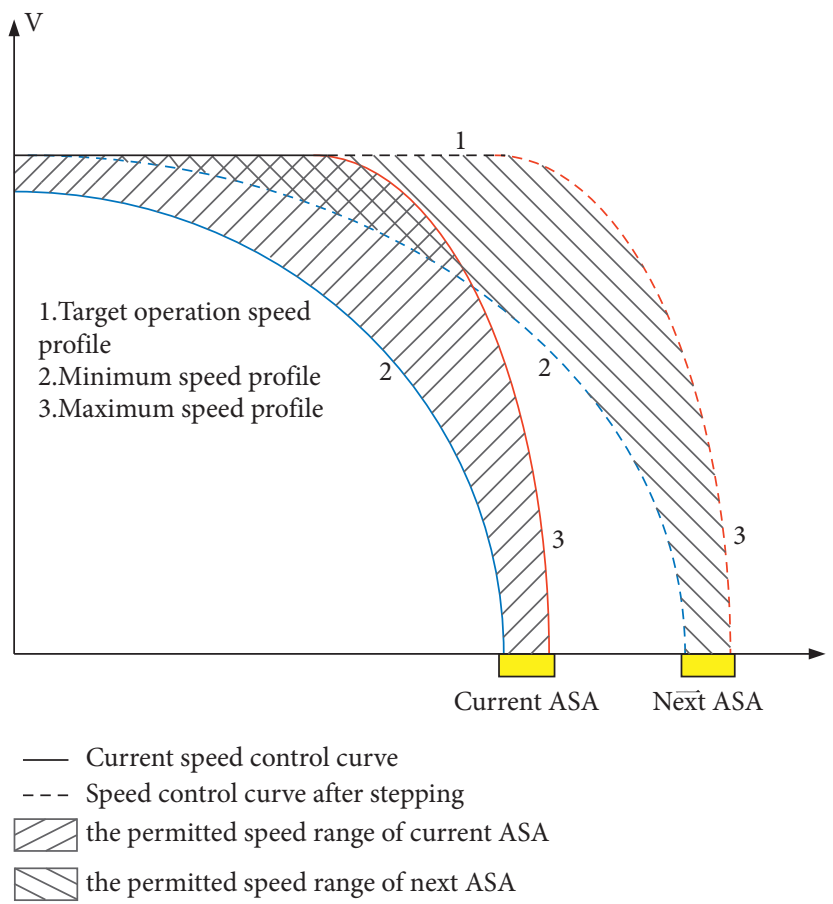

FIGURE 1: The stopping point stepping model for maglev train operation. ASA is the auxiliary stopping area. The speed control curve is the speed limit due to the line and train situation.

The calculation of the safe levitation profile also needs to consider adverse conditions, including the no-load train, the headwinds effect, and the friction coefficient between the track guide-way and the train skid becoming larger. Similarly, the formula for calculating the safe levitation speed profile is

$$
\left\{\begin{array}{l}
v_{t+1}^{l}=v_{t}^{l}+a_{t l}^{w} * \Delta t, \\
s_{t+1}^{l}=s_{t}^{l}+v_{t}^{l} * \Delta t+\frac{a_{t l}^{w} * \Delta t^{2}}{2},
\end{array}\right.
$$

where $v_{t}^{l}$ is the velocity component at the time $t$ of the safety levitation profile; $s_{t}^{l}$ is the position component at the time $t$ of the safety levitation profile; and $a_{t l}^{w}$ is the acceleration of the train at time $t$ under adverse conditions.

There is also a safety margin between the minimum speed profile and the safe levitation profile. According to the system delay $\Delta t_{l}$ from issuing the traction cutoff command to the completion of the traction cutoff, the velocity measurement error $\Delta v$, the positioning error $\Delta s$, and the maximum acceleration of the train $a_{t l}^{w l}$ during time $\Delta t_{l}$, the minimum speed profile can be calculated as

$$
\left\{\begin{array}{l}
v_{t}^{\min }=v_{t}^{l}+a_{t l}^{w l} * \Delta t_{l}+\Delta v, \\
s_{t}^{\min }=s_{t}^{l}-\frac{a_{t l}^{w l} * \Delta t_{l}^{2}}{2}+v_{t}^{\min } * \Delta t_{l}+\Delta s,
\end{array}\right.
$$

where $v_{t}^{\min }$ is the velocity component of the minimum speed profile and $s_{t}^{\min }$ is the position component of the minimum speed profile.
2.2. Optimization Model. Based on the stopping point stepping mode, this paper constructs an optimization model of auxiliary stopping area layout for multiple train operation profiles and single-line two-way operation scenarios. Because the length of the auxiliary stopping area is much smaller compared to the distance between the auxiliary stopping areas, this model divides the entire interstation interval into a set of nonoverlapping candidate auxiliary stopping areas according to the length of the auxiliary stopping area. The section where at least one auxiliary stopping area should be set in the interstation section is set as the expected section.

In the research process of this paper, the optimization problem of auxiliary stopping area layout is regarded as an optimal decision-making problem; that is, a decision is made on the selection of each candidate auxiliary stopping area in the interval between stations. Here, each candidate auxiliary stopping area $i$ includes 6 properties $\left(R P_{i}, H P_{i}, L A_{i}, S A_{i}\right.$, $S C A_{i}$, and $S S A_{i}$ ), respectively, representing the position of the auxiliary stopping area reachable point, the position of the hazard point, length, maximum slope, the changing situation of the slope (1 when it is at the point of changing slope, or 0 otherwise), and spanning-traction section situation ( 1 when crossing two sections, or 0 otherwise). Also, the candidate auxiliary stopping area $i$ belongs to the candidate auxiliary stopping area set $\mathbf{I}$. Each traction section $k$ includes 3 properties $\left(D S_{k}, L D_{k}, D S A_{k}\right)$, which, respectively, represent the range and length of the traction section $k$ and whether there is an auxiliary stopping area in this traction section ( 1 if there is any, 0 otherwise). In addition, the traction section $k$ belongs to the traction section set $\mathbf{K}$. Each expected section $l$ includes 2 properties $\left(R S_{l}, R S A_{l}\right)$, 
which, respectively, represent the range of the $l$ section and whether there is an auxiliary stopping area in this section (1 if there is any, 0 otherwise). In addition, the expected section $l$ belongs to the expected section set $\mathbf{L}$. Meanwhile, the train upwards operation curve set $\mathbf{P}$ and the train downwards operation curve $\mathbf{Q}$ are defined. The train operation curves $p$ and $q$ belong to the set $\mathbf{P}$ and $\mathbf{Q}$.

For single-line two-way operation scenarios, the auxiliary stopping area on the line should meet the continuous stepping of the two-way train requirement. At the same time, the auxiliary stopping area cannot be located in dangerous sections such as steep slopes. In addition, it should be arranged in the sections where there is a demand, such as sections that are convenient for evacuation and sections that can implement a smaller tracking interval. These scene requirements and layout principles can be represented by the above symbols.

First of all, the auxiliary stopping area cannot span the traction sections, which can be written as

$$
\sum_{i} S S A_{i}=0, \forall i \in\left\{i \mid x_{i}=1\right\},
$$

Moreover, the auxiliary stopping area cannot be located at the point of slope change; that is,

$$
\sum_{i} S C A_{i}=0, \forall i \in\left\{i \mid x_{i}=1\right\} .
$$

The slope of the auxiliary stopping area cannot exceed a certain range; that is,

$$
\max \left(\left|S A_{i}\right|\right)-S A_{\max } \leq 0, \forall i \in\left\{i \mid x_{i}=1\right\},
$$

where $S A_{\max }$ is the maximum slope value allowed by the auxiliary stopping area.

Except for the departure station and the terminal station, there should be at least an auxiliary stopping area in each traction section, namely,

$$
\sum_{k} D S A_{k}-N^{d e c}+2=0, \quad \forall k \in\left\{2,3, \ldots, N^{d e c}-1\right\},
$$

where $N^{d e c}$ is the number of traction sections, which includes departure station and terminal station.

At least an auxiliary stopping area is required on the expected section; that is,

$$
\sum_{l} R S A_{l}-N^{r e q}=0, \quad \forall l \in \mathbf{L},
$$

where $N^{r e q}$ is the number of expected sections of the auxiliary stopping area.

The total length of the auxiliary stopping areas is less than a certain value; that is,

$$
\sum_{i} x_{i} L A_{i}-L^{\max } \leq 0, \quad \forall i \in \mathbf{I}
$$

where $L^{\max }$ is the maximum total length of the expected auxiliary stopping areas.

The auxiliary stopping area layout fulfills the conditions of the stopping point stepping of the train bidirectional operation, and there is certain redundancy; that is,

$$
\begin{aligned}
& S T_{\min }-\min \left(S T_{j, j^{+}}^{p}\right) \leq 0, \quad \forall p \in \mathbf{P}, \forall j \in S_{s} \cup i\left|x_{i}=1, \exists j^{+} \in i\right| x_{i}=1 \cup S_{t}, \\
& S T_{\min }-\min \left(S T_{j, j^{+}}^{q}\right) \leq 0, \quad \forall q \in \mathbf{Q}, \forall j \in S_{t} \cup i\left|x_{i}=1, \exists j^{+} \in i\right| x_{i}=1 \cup S_{s},
\end{aligned}
$$

where $S T_{j, j^{+}}^{p}$ is the stepping time for the train to switch from the stop point $j$ to the stop point $j+$ according to the speed profile $p$ [10], that is, the time interval between the intersection of the train running speed profile $p$ and the minimum speed profile of the auxiliary stopping area $j^{+}$and the intersection of this speed profile and the maximum speed profile of the auxiliary stopping area $j ; S T_{j, j^{+}}^{q}$ is the stepping time for the train to switch from the stop point $j$ to the stop point $j^{+}$according to the speed profile $q ; S T_{\min }$ is the minimum required stepping time; and $S_{s}$ and $S_{t}$ represent the departure station and the terminal station, respectively.

The decision variable of this optimization decision problem is $0-1$ variable; that is,

$$
x_{i} \in\{0,1\}, \quad \forall i \in \mathbf{I} \text {. }
$$

For the length of the auxiliary stopping area $L A_{i}$, according to the Shanghai Maglev demonstration line layout experience, combined with the length of the train $L V$, it can be calculated with reference to

$$
L A_{i}= \begin{cases}L V+180 & S A_{i}=0, \\ L V+250 & S A_{i} \leq 0.5 .\end{cases}
$$

The penalty function is often used as a fitness function in the genetic algorithm [18]. In this paper, the method of static penalty function is adopted; that is, the penalty function is only related to whether the solution of the genetic algorithm falls in the feasible region. The optimization model established in this paper has multiple complex constraints, and the penalty value is used to punish the solution that does not meet the constraints so that it can be quickly eliminated in the calculation. Since the maximum value of the objective function $f\left(x_{i}\right)$ is the number of candidate auxiliary stopping areas that can be accommodated by the total length of the maximum 
auxiliary stopping area allowed on the line, a certain number greater than this value can be used as the penalty value. Then, the objective function $F\left(x_{i}\right)$ with penalty terms can be written as

$$
F\left(x_{i}\right)=\left\{\begin{array}{l}
f\left(x_{i}\right), \quad \text { If the constraints are fulfilled, } \\
f_{p}, \quad \text { If any constraint is not fulfilled, }
\end{array}\right.
$$

where $f_{p}$ is a penalty item. When there is a result that does not meet the constraints, $f_{p}$ is taken as a certain number not less than the maximum number of candidate auxiliary stopping areas; otherwise, 0 is taken.

After satisfying the continuous stepping demand of the train and the constraints of some other layout principles on the problem, as a decision, the appropriate section should be selected as the auxiliary stopping area. On the premise of satisfying train operation safety and operational requirements, the economy of the layout plan is considered; that is, the total number of auxiliary stopping areas is as small as possible. Here, the objective function as equation (15) seeks the minimum value of the number of auxiliary stopping areas, which is obtained by accumulating the values of the decision variables:

$$
\min f\left(x_{i}\right)=\sum_{i} x_{i}
$$

where $x_{i}$ is a $0-1$ decision variable. When it is 1 , it means that the $i$ th candidate auxiliary stopping area is selected as the formal auxiliary stopping area. When it is set to 0 , the $i$ th is not selected as the formal auxiliary stopping area.

Therefore, the optimization model can be written as

$$
\left\{\begin{array}{l}
\min f\left(x_{i}\right)=\sum_{i} x_{i}, \\
\text { s.t. } \sum_{i} S S A_{i}=0, \quad \forall i \in\left\{i d \mid x_{i}=1\right\}, \\
\sum_{i} S C A_{i}=0, \quad \forall i \in\left\{i \mid x_{i}=1\right\}, \\
\max \left(\left|S A_{i}\right|\right)-S A_{\max } \leq 0, \quad \forall i \in\left\{i \mid x_{i}=1\right\}, \\
\sum_{k} D S A_{k}-N^{d e c}+2=0, \quad \forall k \in\left\{2,3, \ldots, N^{d e c}-1\right\}, \\
\sum_{l} R S A_{l}-N^{r e q}=0, \quad \forall l \in \mathbf{L}, \\
\sum_{i} x_{i} L A_{i}-L^{\max } \leq 0, \forall i \in \mathbf{I}, \\
S T_{\min }-\min \left(S T_{j, j^{+}}^{p}\right) \leq 0, \quad \forall p \in \mathbf{P}, \forall j \in S_{s} \cup i\left|x_{i}=1, \exists j^{+} \in i\right| x_{i}=1 \cup S_{t}, \\
S T_{\min }-\min \left(S T_{j, j^{+}}^{q}\right) \leq 0, \quad \forall q \in \mathbf{Q}, \forall j \in S_{t} \cup i\left|x_{i}=1, \exists j^{+} \in i\right| x_{i}=1 \cup S_{s} \\
x_{i} \in\{0,1\}, \forall i \in \mathbf{I} .
\end{array}\right.
$$

\section{The Genetic Algorithm for Optimization Model}

3.1. Genetic Algorithm. The genetic algorithm is a reference population evolution mechanism that uses selection, crossover, mutation, replacement, and so on to realize a random search and optimization metaheuristic algorithm. It is often applied to optimization problems, including nonlinear integer optimization problems, to get better results [19].

3.2. Parameter Set. In the algorithm of this paper, the decision variables are stored in vectors form, and binary is used to encode them. This method considers the reference speed profile composed of multiple speed profiles according to the direction of train operation (as shown in equation (17) and Figure 2) [10]:

$$
\left\{\begin{array}{l}
v_{i}^{r}=\min \left(v_{i}^{1}, v_{i}^{2}, \ldots, v_{i}^{n}\right) \\
s_{i}^{r}=s_{i}
\end{array},\right.
$$

where $v_{i}^{r}$ is the speed component of the reference speed profile; $s_{i}^{r}$ and $s_{i}$ are both position components; and $v_{i}^{n}$ represents the speed of the $n$th train speed profile at position $i$.

In terms of population initialization, random methods are often used; that is, the initial population is generated according to a certain distribution function. For binarycoded chromosomes, uniform distribution is often used. In the model designed in this paper, according to the general characteristics "dense low-speed areas and sparse high-speed areas" of the high-speed maglev auxiliary stopping area, an initial population generation method is proposed.

The initial probability $p_{k}^{0}$ is calculated according to the average speed of the section where the candidate auxiliary stopping area is located $\overline{v_{k}}$ and the maximum speed $v_{\max ^{r}}$ of 


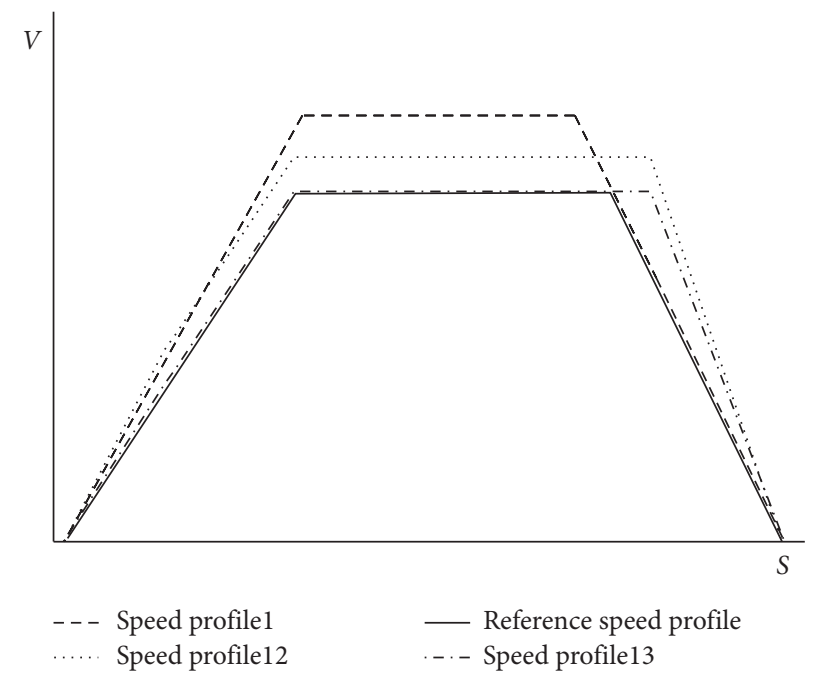

FIgURE 2: The schematic diagram of reference speed profile. The reference speed profile is a combination of multiple speed profiles.

the reference speed curve, and then through normalization, the final probability $P_{k}$ can be obtained, as shown in

$$
\begin{gathered}
p_{k}^{0}=1-\frac{0.75 \overline{v_{k}}}{v_{\text {max }^{r}}} \\
P_{k}=\frac{0.5 p_{k}^{0}}{\max _{k \in K}\left(p_{k}^{0}\right)},
\end{gathered}
$$

The algorithm in this paper selects individuals of the same size from the current population to form mating pools and then obtains a new generation of populations by crossover, mutation, and replacement operators to realize the update of the population, that is, the update of the solution. Moreover, this process ensures the convergence of the solution by memorizing several elite individuals in the current population (i.e., several individuals with the smallest fitness value) and placing them in the next generation population. When a certain number of iterations is met, the individual with the smallest fitness value is output as the solution of the model, that is, the optimal layout of the auxiliary stopping area.

The selection operator used in this article is a conventional roulette strategy. The positions and lengths of the operating points of the crossover and mutation operators are randomly selected, and random crossover and random mutation are performed according to the preset crossover probability and mutation probability. The replacement operator includes the elite individuals into the new generation population. The process is based on the fitness value, replacing several individuals with high fitness values with the elite individuals. The fitness value can be calculated according to the fitness function. The fitness function in this article is the objective function $F\left(x_{i}\right)$ with penalty terms, which is (14).

In summary, the solution flow of the model is listed in Section 3.3.
3.3. Model Solution Steps. This paper considers the method of arranging the optimal layout of the auxiliary stopping area as $0-1$ decision optimization problem, using the singleobjective genetic algorithm to obtain the solution. The specific calculation process is shown in Figure 3; that is, the abstract model is optimized and solved through the following five steps.

Step 1: data preprocessing. According to the condition of the line and the traction section, the expected section is selected where the auxiliary stopping area is expected to be set up, involving the section for evacuation and the section to guarantee the tracking interval, and we divide the entire interstation section into nonoverlapping candidate auxiliary stopping area set, based on the length of the candidate auxiliary stopping areas. Then, properties of each auxiliary stopping area are defined, including the position of the auxiliary stopping area reachable point, the position of the hazard point, length, maximum slope, the changing situation of the slope, and spanning-traction section situation. The reachable point position and the hazard point position are the head-end and the end of the auxiliary stopping area, respectively. Moreover, the reference speed curve is obtained according to the multiple target speed curves of trains. The probability of each candidate auxiliary stopping area being selected is calculated according to the initial population generation method mentioned in Section 3.2.

Step 2: parameters and population initialization. Load the data of line slope, train length and weight, environmental wind speed, and train operation speed profile; initialize the parameters in the optimization model and solution algorithm, including constraint information, crossover probability, mutation probability, genetic iteration number, population size, and elite parameters; and initialize the population. 




FIGURE 3: The simplified flowchart of solving algorithm.

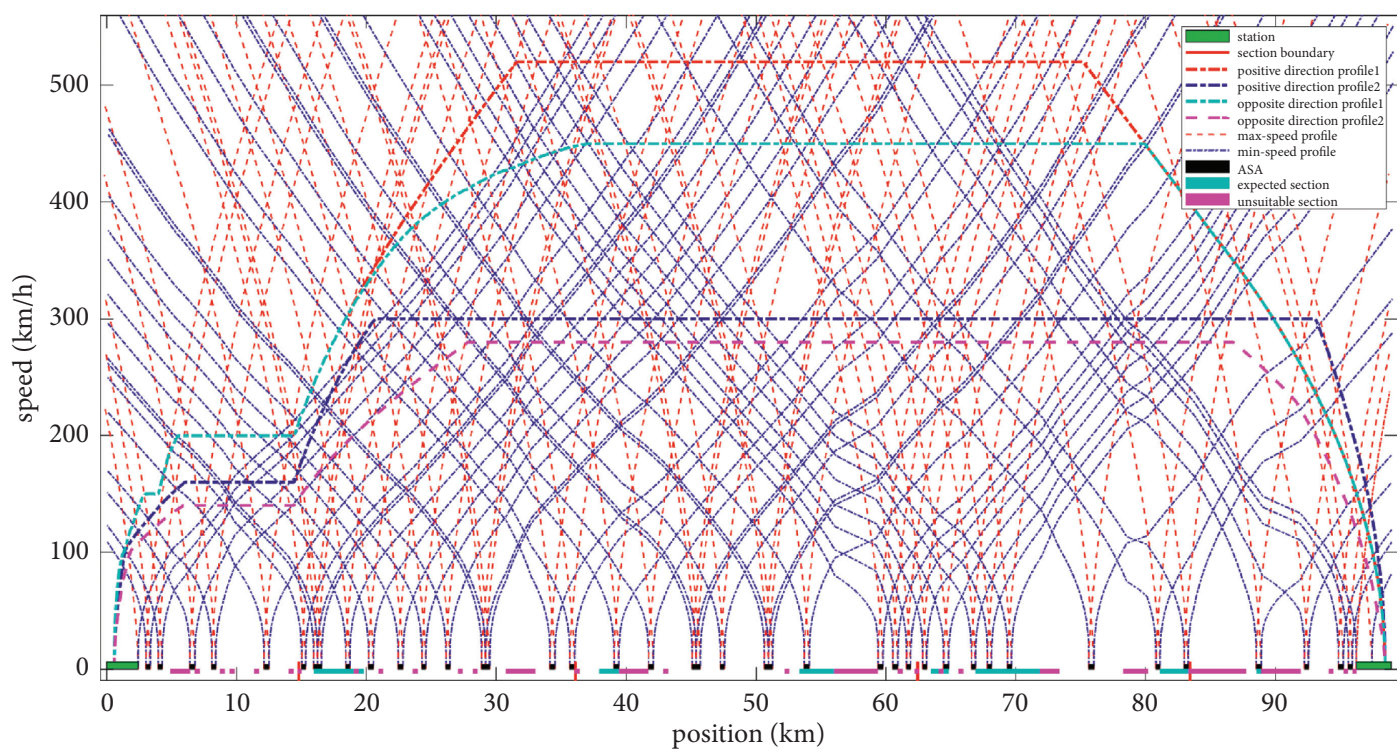

FIgURE 4: Result based on the uniform distribution (stepping redundancy time is $2 \mathrm{~s}$ ). Unsuitable areas are candidate auxiliary stopping areas that do not meet the constraint conditions as equations (5), (6), and (7). The positive direction profiles and the opposite direction profiles are the trains operating speed curves with different directions and reference speeds. The max-speed profile and the min-speed profile are the limitation speed curve of the corresponding auxiliary stopping area.

Step 3: evaluation of the plan. The information of individuals in the population is decoded, and the original objective function value and the minimum stopping point stepping time, the total length of the stopping area, and other constraint information are calculated, respectively, and then the fitness value of each individual is obtained according to the objective function with penalty items. 


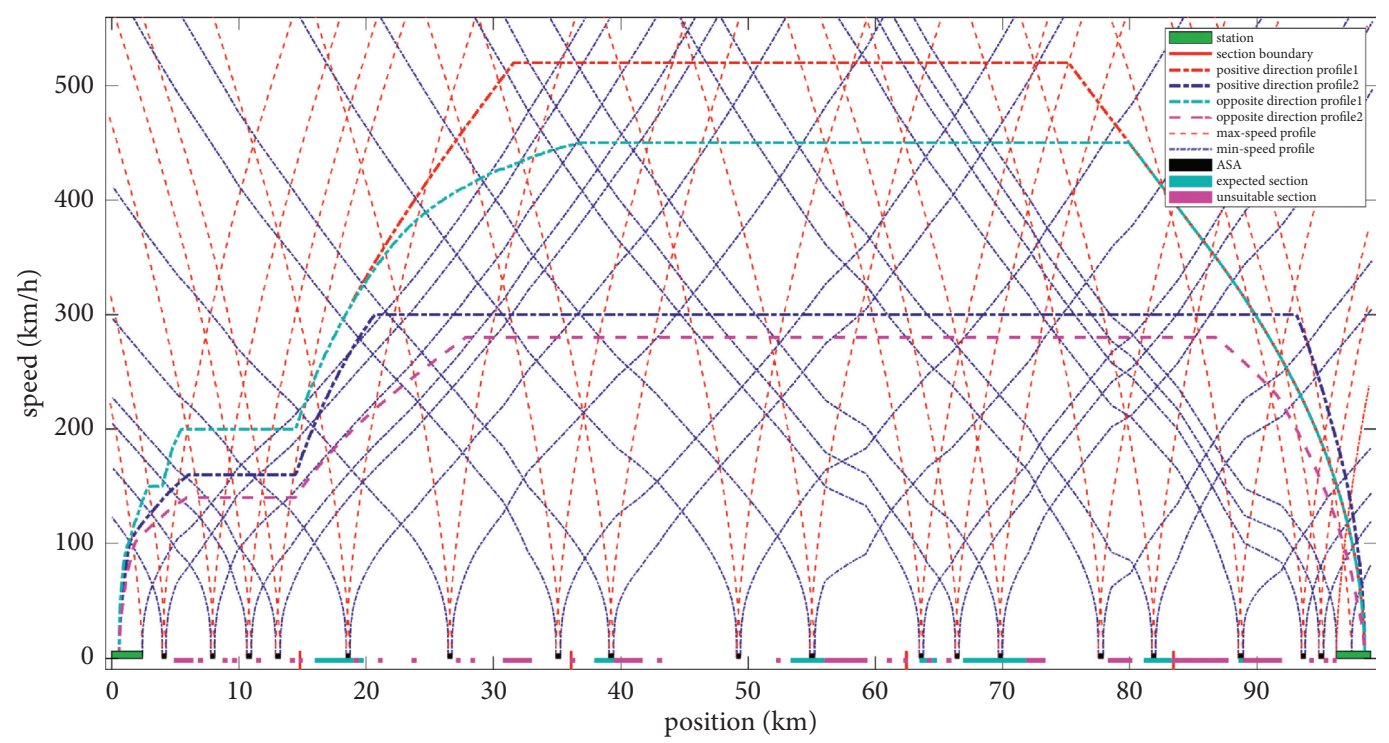

FIGURE 5: Result based on the optimized distribution (stepping redundancy time is $2 \mathrm{~s}$ ).

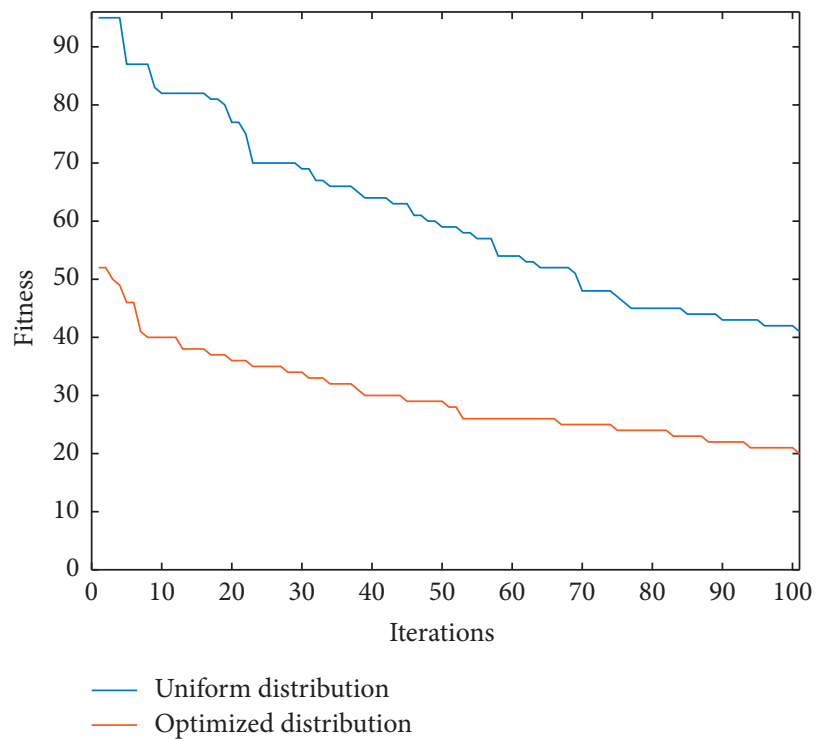

Figure 6: Comparison of iterative curves of different distribution methods. The data in the chart is acquired after averaging the 30 times of running.

Step 4: update population. Based on the fitness value of the individual and the chromosome code, operations such as selection, crossover, mutation, fitness calculation, and replacement are performed to obtain a new population, that is, a new solution.

Step 5: the judgement of terminating optimization. Determine whether the preset termination condition is reached. If reached, output the best individual in the current population as the optimal solution. Otherwise, return to step 3 .

\section{Experiment Results}

This paper analyzes the method's effectiveness in combination with a numerical experiment. The total length of the line involved is $98,900 \mathrm{~m}$, and there are 5 interstation sections. Combining the line slope, train operation safety requirements, and tracking efficiency requirements, there are 7 expected sections. There are two operating speed profiles involved each way respectively of the bidirectional operation trains; the maximum operating speeds of one way are $520 \mathrm{~km} / \mathrm{h}$ and $300 \mathrm{~km} / \mathrm{h}$; and the maximum speeds of the opposite way are $450 \mathrm{~km} / \mathrm{h}$ and $280 \mathrm{~km} / \mathrm{h}$. The crossover probability and mutation probability are taken as 0.8 and 0.2 , respectively; the number of elite groups is 0.1 times the population size; and both the population size and the number of iterations are 100 . According to the population initialization method mentioned in Section 3.2, the probabilities that the candidate auxiliary stopping area in the interstation sections is selected as the formal auxiliary 


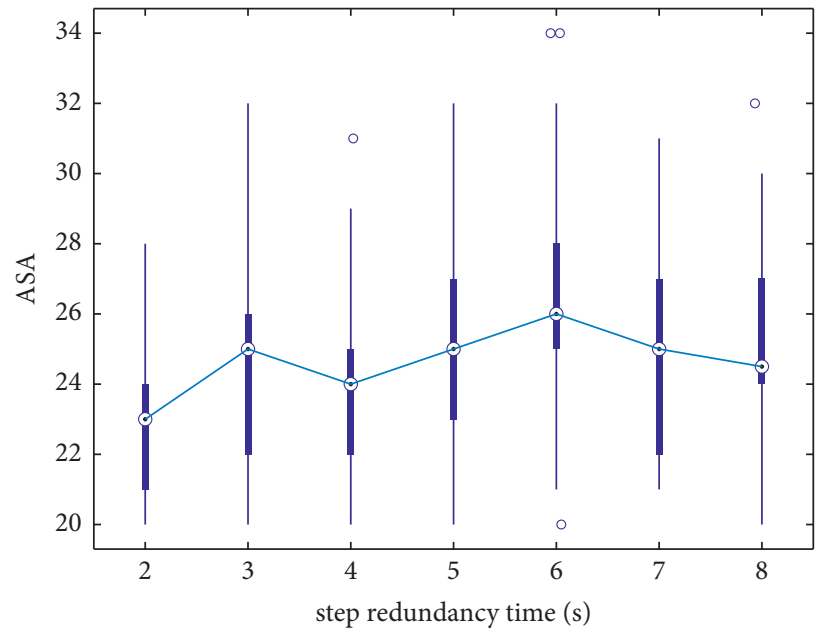

Figure 7: The influence of stepping redundancy time on the demand of auxiliary stopping area. The line connects the median value of the number of ASA on each redundancy time. The thin blue line represents the total value range that 30-time results appear not considered outliers; the bottom and top edges of the thick blue line indicate the $25^{\text {th }}$ and $75^{\text {th }}$ percentiles, respectively; and the outliers are plotted individually using the " $o$ " symbol.

stopping area are $0.500,0.288,0.195,0.195$, and 0.361 , respectively. If a uniform distribution is used, these probabilities are all 0.5 . The penalty value is set to 95 , which is more than the maximum number of auxiliary stopping areas that can be covered by the maximum total length of the auxiliary stopping area.

When the stepping redundancy is set to $2 \mathrm{~s}$, the result of calculation using uniform distribution to generate the initial population randomly is shown in Figure 4, and the result of calculation using this optimization method is shown in Figure 5.

The comparison of the two figures shows that, in the case of the same number of iterations (100 times), the initialization strategy combined with the actual characteristics can make the number of calculated auxiliary stopping areas less, and the distribution is more uniform. Furthermore, the calculation time of Figure 4 is 54.3476 s, and the calculation time of Figure 5 is $33.9115 \mathrm{~s}$.

The iterative calculation result is repeated thirty times, and then the average of the fitness function of each generation in the thirty calculations is taken. The iterative function in Figure 6 shows that the optimized population initialization strategy makes the function converge faster.

This article sets up multiple sets of stepping redundancy time and compares the optimization results. When the stepping redundancy time gradually increases, Figure 7 can be obtained. It can be seen from Figure 7 that the average number of auxiliary stopping areas required in general increases with the increase of the stepping redundancy time, but the optimized results are within a certain range, which shows that we may improve the stepping redundancy time under the limit of the number of auxiliary stopping areas by optimizing the layout. If the stepping redundancy time exceeds $10 \mathrm{~s}$, the layout cannot satisfy the constraints. The current high-speed maglev system stepping redundancy time has no clear requirements, but the design time usually assumes the stepping can be completed around 0.2 seconds.
This paper considers complicated line situations, multiple train operation speed profiles, and two-way operation scenarios, so the method in this paper has better versatility than previous studies.

\section{Conclusion}

High-speed maglev lines may have scenarios such as bidirectional operation on single-track, multiple train operation speed profiles, expected sections, and complicated line situations. Previous studies are difficult to meet the safety and efficiency requirements of this situation. This paper proposes a method of high-speed maglev auxiliary stopping area layout that is suitable for this scenario. The numerical experiment results show that the layout scheme proposed by this method meets the requirements of safety operation and scenes of trains and has high feasibility and universality. In addition, the distribution of the output of the population initialization method proposed in this paper can effectively improve the calculation efficiency. The next step of the research can focus on optimizing the auxiliary stopping area layout when the number of auxiliary stopping areas is limited.

\section{Data Availability}

The data used to support the findings of this study are available from the corresponding author upon request.

\section{Conflicts of Interest}

The authors declare that there are no conflicts of interest regarding the publication of this study.

\section{Acknowledgments}

This work was jointly supported by the National Key R\&D Program of China (Grant no. 2016YFB1200602-02), the 
Science Research Project of Shanghai Science and Technology Committee (Grant no. 18DZ1205803), the Foundation of Shanghai Maglev and Rail Transit Cooperative Innovation Center (Grant no. 20132223), and the R\&D Project of Shanghai Shentong Metro Group Co., Ltd. (JSKY19R012-WT-20018).

\section{References}

[1] W. W. Dickhart, "Status of transrapid maglev," in Proceedings of the Electro International 1991, pp. 613-617, IEEE, New York, NY, USA, 1991.

[2] R. Schach, J. Peter, and R. Naumann, Transrapid und RadSchiene-Hochgeschwindigkeitsbahn, Springer, Berlin, Germany, 2006.

[3] X. Wu, Maglev Train, Shanghai Scientific \& Technical Publishers, Shanghai, China, 2003.

[4] G. Yang and Z. Tang, "The analysis of high-speed wheel-rail train and high-speed maglev train safety systems," in Proceedings of ICSSSM '05. International Conference on Services Systems and Services Management, pp. 1403-1407, Chongquing, China, 2005.

[5] F. Chen and $\mathrm{H}$. Xu, "Research on speed protection curve of German high-speed maglev transportation," China Science and Technology Information, vol. 17, pp. 248-251, 2006.

[6] Ya Jiang, W. Wu, and J. Liu, "Simulation of high-speed maglev train 2-d speed protection curve," Journal of Tongji University, vol. 32, no. 3, pp. 397-400, 2004.

[7] Q. Lai, J. Liu, H. Ali, L. Meng, and Y. Wang, "Energy-efficient speed profile optimization for medium-speed maglev trains," Transportation Research Part E: Logistics and Transportation Review, vol. 141, Article ID 102007, 2020.

[8] J. Liu and W. Wu, "Research on 2-d speed protection curve and its algorithm of high-speed maglev transportation," China Railway Science, vol. 23, no. 4, pp. 106-110, 2002.

[9] G. Yang and Z. Tang, "Study on safety speed protection of the high maglev train," Journal of Beijing Jiaotong University, vol. 31, no. 2, pp. 38-42, 2007.

[10] Y. Yi, X. Jiang, H. Lin, and H. Zhao, "Setting method of auxiliary stopping area for high-speed maglev based on protection speed," Journal of Tongji University, vol. 47, no. 9, pp. 1310-1316, 2019.

[11] Z. Zhang, Y. Liu, and Y. Feng, "Study and simulation of maglev railway tracing model," Journal of System Simulation, vol. 4, pp. 841-846, 2006.

[12] J. Bian, Research on Properties of Maglev Train Operation Control System and Characteristics of Auxiliary Stopping Aarea, Zhejiang University, Zhejiang, China, 2006.

[13] Yu Yi, P. Chu, D. Dong, Xi Jiang, H. Zhao, and H. Lin, "Auxiliary stopping area layout method for high-speed maglev operated bidirectionally on single track," Journal of Advanced Transportation, vol. 2021, Article ID 5571788, 15 pages, 2021.

[14] Xi Jiang, Yu Yi, Z. Deng, H. Lin, G. Chen, and H. Zou, "Setting optimization of auxiliary stopping area for high-speed maglev considering train tracking operation," Journal of Railway Science and Engineering, vol. 18, no. 2, pp. 325-333, 2021.

[15] Q. Lai, Q. Lai, J. Liu et al., "Optimization of the auxiliary stopping area planning in the middle-to-high speed maglev," Transportation Systems and Technology, vol. 4, no. 2, pp. 141-151, 2018.

[16] Y. Tian, S. Geng, Y. Wang, J. Xue, and L. Chen, "Placement of the assist stop areas in high-speed maglev system considering bidirectional operation," Journal of Physics: Conference Series, vol. 1622, no. 1, Article ID 012128, 2020.
[17] H. Zhao, Y. Chen, Z. Liao, and Y. Yi, "Study of speed curve monitoring technology for a high-speed maglev train operation," in Proceedings of 16th COTA International Conference of Transportation Professionals, Shanghai, China, 2016.

[18] Z. Meng, M. Jiang, and C. Dang, "Evolutionary algorithm for zero-one constrained optimization problems based on objective penalty function," in Proceedings of 2010 International Conference on Computational Intelligence and Security, pp. 132-136, Nanning, China, 2010.

[19] M. Sakawa, K. Kato, Md.A. K. Azad, and R. Watanabe, “A genetic algorithm with double string for nonlinear integer programming problems," in Proceedings of 2005 IEEE International Conference on Systems, Man and Cybernetics, vol. 4, pp. 3281-3286, Toronto, ON, Canada, 2005. 